\title{
Construction of kernels for nonlocal elasticity from one-dimensional dispersion data in reciprocal space
}

\author{
S. Ghosh* $\quad$ V. Sundararaghavan ${ }^{\dagger}$ \\ A.M. Waas ${ }^{\ddagger}$ \\ Department of Aerospace Engineering, University of Michigan, Ann Arbor, MI, USA
}

\begin{abstract}
Kernels for non-local elasticity are frequently obtained from phonon dispersion relations. The dispersion relations are generally available in the form of one-dimensional (1D) curves in reciprocal space for different high-symmetry directions of the Brillouin zone, however, 3D kernels for three-dimensional (3D) solids are needed. To the best of knowledge of the authors, there is no systematic procedure to obtain material specific nonlocal kernels (for integral type nonlocality) from the dispersion data. The present paper proposes strategies to build the 3D kernels from the dispersion data. Our particular focus is on isotropic media that is interesting due to the inherent quasi-1D nature of the axisymmetric kernel. We have obtained these kernels using Fourier-Bessel transforms, yielding axisymmetric kernel profiles in both reciprocal and real spaces.
\end{abstract}

\section{Introduction}

Theories of classical continuum mechanics do not account for length scales that capture the underlying material microstructure. Consequently, they are found to be inadequate for several situations, where microstructure can influence the deformation response in a manner that is not adequately captured by classical continuum mechanics. Some examples include crack-tip stress states, dispersive propagation of waves, strain softening etc. Nonlocal continuum theories can alleviate the aforementioned problems, if a suitable length scale parameter can be found which is representative of the underlying material microstructure. Furthermore, meso-scale modeling, which is needed to bridge the gap between atomistic models and their upscaled continuum models, will necessarilly have to address the issue of incompatibility between atomistic models which are non-local and contain length scales and classical continnum models which are devoid of a length scale. The results from several prior studies on non-local theories can be found in, Bazant and Jirásek

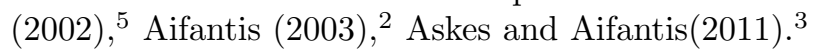

In the integral type nonlocal theories, the stress is related to a weighted integral of strains of over a certain neighborhood. The weighting function is the so called kernel. The nonlocal stress, $t_{k l}$, in linear elastic body, $V$, can be described as

$$
t_{k l}(\boldsymbol{x})=\int_{\Omega} \alpha_{i j k l}\left(\boldsymbol{x}, \boldsymbol{x}^{\prime}\right) \epsilon_{k l}\left(\boldsymbol{x}^{\prime}\right) d \Omega
$$

where $\boldsymbol{\alpha}$ is a tensorial kernel representing an attenuating elastic modulus. Here, $\boldsymbol{t}$ and $\boldsymbol{\epsilon}$ are the nonlocal stress tensor and local strain respectively. $\Omega \subset V$ is the compact support for the kernel. $\boldsymbol{x}$ and $\boldsymbol{x}^{\prime}$ are position vectors for two material points in $\Omega$. If it is assumed that a unique kernel weights all entries of the stiffness tensor, then the above equation becomes,

$$
t_{k l}(\boldsymbol{x})=\int_{\Omega} \alpha\left(\boldsymbol{x}, \boldsymbol{x}^{\prime}\right) \sigma_{k l}\left(\boldsymbol{x}^{\prime}\right) d \Omega
$$

\footnotetext{
*Postdoctoral Scholar of Aerospace Engineering, Email: susantag@umich.edu

${ }^{\dagger}$ Assistant Professor of Aerospace Engineering, Email: veeras@umich.edu, AIAA Member. Corresponding and presenting author

${ }^{\ddagger}$ Felix Pawlowski Collegiate Professor of Aerospace Engineering; visiting Professor, Department of Aeronautics, Imperial College, London, Email: dcw@umich.edu, AIAA Fellow
} 
Here, $\boldsymbol{\sigma}$ is the Hookean stress tensor and $\alpha$ is a scalar kernel function. When the kernel function, $\alpha$, tends to a delta function the nonlocal elasticity degenerates to the classical elasticity. The material dependence of the nonlocal constitutive equations appears yet to be resolved, nevertheless the representation provided in equation (2) is widely used and the following properties are generally attributed to the Kernel function, $\alpha$ :

(1) $\alpha\left(\boldsymbol{x}, \boldsymbol{x}^{\prime}\right)$ satisfies the normalization condition, i.e. $\int_{\Omega} \alpha\left(\boldsymbol{x}, \boldsymbol{x}^{\prime}\right) d \Omega=1$

(2) The kernel is a decaying function i.e. it has peak at $\left\|\boldsymbol{x}-\boldsymbol{x}^{\prime}\right\|=0$, decay with increasing distance $\left\|\boldsymbol{x}-\boldsymbol{x}^{\prime}\right\|$ and stays positive in $\Omega$.

(3) $\alpha$ is bi-symmetric i.e. $\alpha\left(\boldsymbol{x}, \boldsymbol{x}^{\prime}\right)=\alpha\left(\boldsymbol{x}^{\prime}, \boldsymbol{x}\right)$ and function of $\boldsymbol{x}-\boldsymbol{x}^{\prime}$. For isotropic materials $\alpha$ is axisymmetric (direction independent), i.e. $\alpha\left(\boldsymbol{x}, \boldsymbol{x}^{\prime}\right)=\alpha(r)$, where $r=\left\|\boldsymbol{x}-\boldsymbol{x}^{\prime}\right\|$.

For detailed description about the properties of the kernel function refer to, Eringen(1983), ${ }^{7}$ Bazant and Chang(1984) $\stackrel{[4}{,}$ Bazant and Jirásek(2002) ${ }^{[5}$ Polizzotto(2001) ${ }^{11}$. Perhaps the kernel function, should also be able to represent a delta function as $\Omega \rightarrow 0$, as suggested in Eringen $(1983)^{7}$. In this context ${ }^{4}$ suggested that a continuum should not yield zero energy mode for non-rigid-body deformations and should have real wave propagation velocity. They have shown that these two conditions requires Fourier transform of $\alpha$ to have positive values that span the entire reciprocal space. Same restriction on $\alpha$ was reached in Polizzotto(2001) $)^{11}$ by noting that the equation 2 is a homogeneous Fredholm integral equation of first kind and then invoking the Fredholm integral equation theory. It was noted in Bazant and Chang(1984) $)^{4}$ that some of the popular kernels do not satisfy the required conditions, they have suggested to include delta function alleviate this problem. However, we point out that inclusion of delta function in the kernel would invariably lead to the lose of stress regularity property of nonlocal elasticity whenever the local stress is singular.

On contrary to the above mentioned restrictions on the kernel, recent research through molecular mechanics furnishes numerical results which indicate that at nano-scale, $\alpha$ may not be always positive and the attenuation need not be monotonous $10[13$ It appears that the negative values in the nano-scopic $\alpha$ could be an artifact of empirical analytical inter-atomic potential used in these studies. In Picu (2002) $\frac{10}{10}$ using the discrete atomistic interactions between randomly distributed atoms analytical kernels were derived for isotropic materials. Therein the $1 \mathrm{D}$ kernel is obtained from molecular simulation in 3D, containing contributions form the neighbouring volume, which is different form 1D kernel obtained from 1D or quasi-1D system. Subsequently the 3D kernel is obtained from the 1D kernel by equating the nonlocal stress at the centre of a 3D domain, which apparently lacks physical basis. Further, their 3D kernels are not defined for distances below the distance at which the radial distribution function goes to zero.

Dispersion curves are obtained for different modes of waves propagation along various wave vectors. The dispersion curves obtained in this manner are inherently one dimensional in nature, whereas for analyzing continuum (represented via integral type nonlocality) 3D kernels are needed. With best of authors' knowledge there does not exist systematic procedure to obtain material specific nonlocal kernels (for integral type nonlocality) from dispersion data. For ideal isotropic materials the longitudinal and transverse wave dispersion curves should be identical upon proper scaling by phase velocities of classical elasticity, consequently the kernels obtained for different modes would be identical. Present paper provides methods for obtaining the $3 \mathrm{D}$ kernel form the frequency dispersion data.

\section{Construction of 3D kernels}

The problem addressed below is that given $\hat{\alpha}_{1 D}(k)$, where $k$ is the wave number, the $\alpha_{3 D}(\boldsymbol{x})$ has to be found. Note that if a function $f(\boldsymbol{x})$ defined on a n-D domain can be written as a tensor product of $n$ functions (along orthogonal directions) defined over real line, as $f(\boldsymbol{x})=f_{1}\left(x_{1}\right) \otimes f_{2}\left(x_{2}\right) \otimes \cdots \otimes f_{n}\left(x_{n}\right)$ the Fourier transform can be simplified as 9

$$
\mathcal{F}(f(\boldsymbol{x}))=\mathcal{F}\left(f_{1}\right) \otimes \mathcal{F}\left(f_{2}\right) \otimes \cdots \otimes \mathcal{F}\left(f_{n}\right)
$$


This kind of functions are called as separable functions. Therefore the kernel in real space, $\alpha_{1 D}(x)$, can be obtained as tensor product of inverse Fourier transform of $\hat{\alpha}_{1 D}(k)$ 's along different axes of the Cartesian coordinates.

$$
\alpha_{3 D}(\boldsymbol{x})=\alpha_{1 D}^{x_{1}}\left(x_{1}\right) \alpha_{1 D}^{x_{2}}\left(x_{2}\right) \alpha_{1 D}^{x_{3}}\left(x_{3}\right)
$$

This kind of construction of multi-dimensional kernel may be suitable for orthotropic case. However, they are not suitable for isotropic case in general as discussed in the following.

Focus of the present work is on isotropic materials, for which the $\alpha_{2 D}(\boldsymbol{x})$ and $\alpha_{3 D}(\boldsymbol{x})$ should have cylindrical and spherical symmetries respectively (i.e. rotational symmetry in 2D and 3D respectively). For isotropic materials the tensor product scheme may not work, since functions with rotational symmetry may not be separable. However, there are exceptions, for instance Gaussian functions are axisymmetric as well as separable. It is know that every circularly symmetric separable function are Gaussian, $\frac{12}{, 2}$. For separable axisymmeric functions the multidimensional kernels can be constructed via tensor product as described for anisotropic case. The following part of this section focuses on kernel construction for axisymmetric functions. In case of rotational symmetry of $\alpha_{n D}(\boldsymbol{x})$ its Fourier transform $\hat{\alpha}_{n D}(\boldsymbol{k})$ proves also to be rotationally symmetrical. Here, $\boldsymbol{k}$ is the position vector in the reciprocal space (wave vector). In $\mathrm{nD}$ the circular symmetry for a function $f$ means $f\left(x_{1}, x_{2}, \cdots, x_{n}\right)=f^{r}(r)$ where, $r=\left(x_{1}^{2}+x_{2}^{2}+\cdots, x_{n}^{2}\right)^{1 / 2}$ The superscript ' $\mathrm{r}$ ' refers to radial function. Note that $f$ and $f^{r}$ are different functions. Its Fourier transform $\hat{f}\left(k_{1}, k_{2}+\cdots, k_{n}\right)$ is also circularly symmetric in $\boldsymbol{k}$-space. That is the n-D Fourier transform of a n-D circularly symmetric function is circularly symmetric. It turns out that for 2 - dimension the radial profile of the Fourier transformed function is identical to the Hankel transform ${ }^{6}$ of zero order of the radial profile (in the interval $0 \leq r<\infty$ ) of the 2-D circularly symmetric function. The relation between these two radial functions is obtained by Hankel transform, ${ }^{6}$ also known as Fourier-Bessel transform, (here denoted as $\mathcal{H}_{0}$, see equation 5). Similarly for 3D axisymmetric functions the relation between the two radial functions is obtained by the spherical Bessel transform ${ }^{6}$ of zero order (here denoted as $\mathcal{S}_{0}$, see equation 6). These particular properties of the Fourier transform for $\mathrm{nD}$ axisymmetric functions is the key for the current paper.

\section{A. Kernel construction using known radial profile}

In view of the rotational symmetry of the kernels in higher dimension, two different routes for the construction of the kernel are explored in the following. While the first approach assumes the radial profiles are identical for different dimensions in the "reciprocal space", the second approach assumes the same but for "real space". It will be clear subsequently that kernels obtained on the basis of these two approaches are different in general.

\section{First approach}

The first approach assumes:

$$
\hat{\alpha}_{n D}^{r}(k)=c_{n D} \hat{\alpha}_{1 D}(k), \quad n=2,3
$$

Therefore,

$$
\begin{aligned}
& \alpha_{2 D}^{r}(r)=c_{2} \mathcal{H}_{0}^{-1}\left(\hat{\alpha}_{1 D}(k)\right)=\frac{c_{2}}{2 \pi} \int_{0}^{\infty} \hat{\alpha}_{1 D}(k) J_{0}(k r) k d k \\
& \alpha_{3 D}^{r}(r)=c_{3} \mathcal{S}_{0}^{-1}\left(\hat{\alpha}_{1 D}(k)\right)=\frac{c_{3}}{2 \pi^{2}} \int_{0}^{\infty} \hat{\alpha}_{1 D}(k) \operatorname{sinc}(k r) k^{2} d k
\end{aligned}
$$

Where, the constants $c_{2}$ and $c_{3}$ are used to satisfy the normalization condition.

In the following the constants $c_{2}$ and $c_{3}$ are obtain using the normalization conditions

$$
\begin{aligned}
& 2 \int_{0}^{\infty} \alpha_{1 D}^{r}(r) d r=1 \\
& \int_{0}^{2 \pi} \int_{0}^{\infty} \alpha_{2 D}^{r}(r) r d r d \theta=1 \\
& \int_{0}^{2 \pi} \int_{0}^{\pi} \int_{0}^{\infty} \alpha_{3 D}^{r}(r) r^{2} \sin (\phi) d r d \phi d \theta=1
\end{aligned}
$$

These constants can be obtained using orthogonality property of Bessel and Spherical Bessel functions, see Ghosh, Sundararaghavan and Waas (2013) 8 for details. 


\section{Second approach}

The second approach assumes: $\alpha_{n D}^{r}(r)=C_{n D} \alpha_{1 D}^{r}(r), n=2,3$. Therefore in this approach the intended kernel in real space is obtained more directly. The normalization condition for the kernels as given by equation (8) and $(9)$ yield the $C_{n D}$-s as $C_{2 D}=\frac{1}{2 \pi I_{2}}$, where, $I_{2}=\int_{0}^{\infty} \alpha_{1 D}(r) r d r$ and $C_{3 D}=\frac{1}{4 \pi I_{3}}$, where, $I_{3}=$ $\int_{0}^{\infty} \alpha_{1 D}(r) r^{2} d r$.
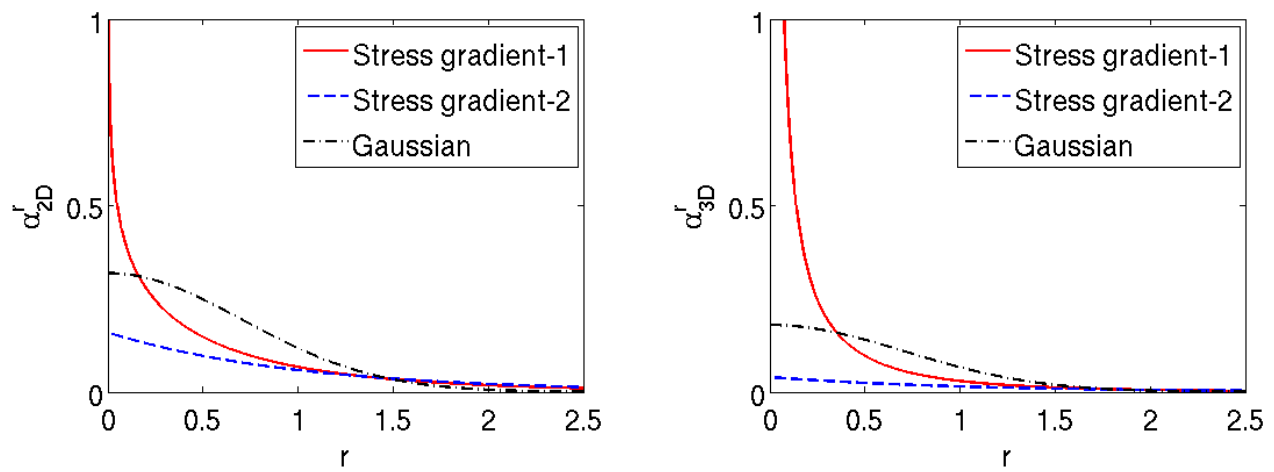

Figure 1. Radial profile for 2D and 3D Kernels. For all kernels $c=1$.

\section{Examples}

In this section few commonly used functional form of 1D kernels were explored to obtain the axisymmetric $2 \mathrm{D}$ and $3 \mathrm{D}$ kernels.

\section{A. Isotropic non-separable kernel: stress gradient}

The $1 \mathrm{D}$ kernel $\hat{\alpha}_{1 D}=1 /\left(1+c^{2} k_{1}^{2}\right)$ corresponds to the to the stress gradient theory. According to the first approach the radial functions in reciprocal space in $1 \mathrm{D}$ to $3 \mathrm{D}$ is given by $\hat{\alpha}_{n D}=1 /\left(1+c^{2} k^{2}\right)$, where, $k=\|\boldsymbol{k}\|, \boldsymbol{k} \in \mathbb{R}^{n}$. Following the first approach the radial function for the kernels in the real space are

$$
\alpha_{1 D}\left(x_{1}\right)=\frac{1}{2 c} e^{-\left|x_{1}\right| / c}, \quad \alpha_{2 D}^{r}(r)=\frac{1}{2 \pi c^{2}} K_{0}(r / c), \quad \alpha_{3 D}^{r}(r)=\frac{1}{4 \pi c^{2} r} e^{-r / c}
$$

Where, $x_{1} \in \mathbb{R} ; r=\|\boldsymbol{x}\|, \boldsymbol{x} \in \mathbb{R}^{n}, n=2,3$. Note that $c>0$ is a constant and $K_{0}$ is the modified Bessel Function of the Second Kind of order zero, see page-376 of Abramowitz and Stegun(1972)! 1 The multi dimensional kernels shows singularity at $r=0$. Note that these are the Green's function for the operator $1-c^{2} \nabla^{2}$ in 1,2 and 3 dimensions. Therefore, kernels obtained following the first approach corresponds to the Green's function of the stress gradient differential operator. The 1D kernel has jump discontinuity of unit magnitude like Heaviside function in its derivative at origin. The 2D and 3D kernels have (essential and pole respectively) singularity at origin.

Following the second approach the radial function for the kernels in the real space are

$$
\alpha_{2 D}^{r}(r)=\frac{1}{2 \pi c^{2}} e^{-r / c}, \quad \alpha_{3 D}^{r}(r)=\frac{1}{8 \pi c^{3}} e^{-r / c}
$$

Where, $r=\|\boldsymbol{x}\|, \boldsymbol{x} \in \mathbb{R}^{n}, n=2,3$. Therefore, the two approaches yield completely different kernels in the real space. While the first approach yields only one singularity at $r=0$ the second approach does not have any singularity.

\section{B. Isotropic separable kernel: Gaussian}

The 1D Gaussian kernel in reciprocal space $e^{-c^{2} k^{2} / 4}$. If the coefficient $c$ is same along different Cartesian axes then this is a separable function. For separable axisymmetric function construction using tensor product 

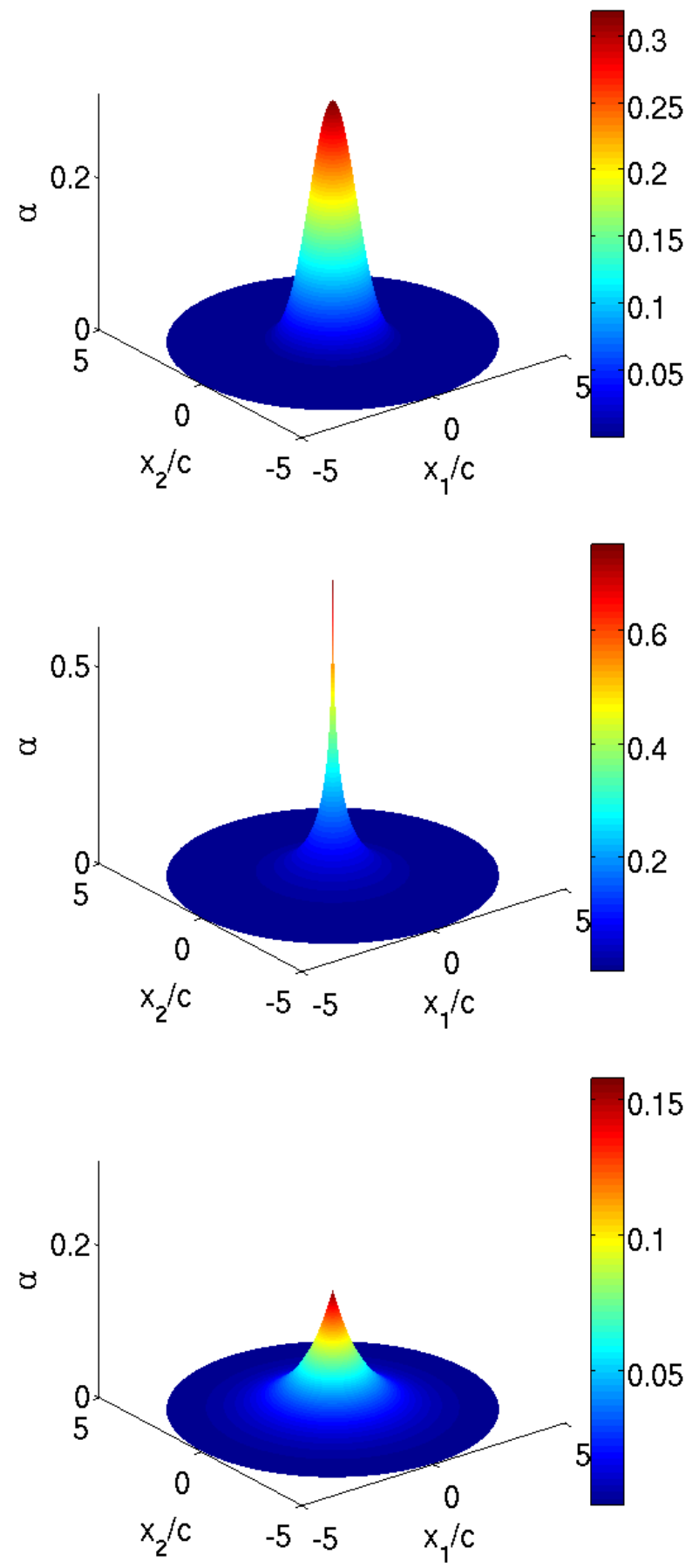

Figure 2. 2D kernels $(c=1)$. Gaussian, stress gradient 1st and 2nd approach from left to right. 
approach and radial function approach will yield identical kernels. Following the first approach the radial function for the kernels in the real space are

$$
\alpha_{1 D}\left(x_{1}\right)=\frac{e^{-\frac{r^{2}}{c^{2}}}}{c \sqrt{\pi}}, \quad \alpha_{2 D}^{r}(r)=\frac{e^{-\frac{r^{2}}{c^{2}}}}{c^{2} \pi}, \quad \alpha_{3 D}^{r}(r)=\frac{e^{-\frac{r^{2}}{c^{2}}}}{c^{3} \pi^{3 / 2}}
$$

where, $x_{1} \in \mathbb{R}, r=\|\boldsymbol{x}\|, \boldsymbol{x} \in \mathbb{R}^{n}, n=2,3$. The 2D and 3D kernels obtained by second approach is identical to the above.

The radial profiles for kernels obtained by both of the proposed approaches are compared in figure (1), which shows that kernel properties depend on the choice of construction proposed above and also the kernels depend on the dimension of the problem. Surface plots for 2D kernels as given in figure (2) emphasizes the same.

\section{Conclusion}

This paper provides a general approach to obtain isotropic multi-dimensional kernels, useful for nonlocal elasticity, from phonon dispersion data. The key issue here is that the kernels obtained must be axisymmetric functions in both real as well as in reciprocal space due to isotropy. In order to satisfy the axisymmetry two different techniques (namely first and second approach respectively) are proposed. Multi-dimensional axisymmetric kernels built in this manner have identical radial profile with the 1D kernel in either, the (1) reciprocal space or (2) real space. For the first approach, the kernels are obtained as the generalized Hankel transform of the radial profile of the kernels in reciprocal space. Multi-dimensional kernels obtained via both approaches are normalized. Some example multi-dimensional analytical kernels are developed to demonstrate the method.

\section{Acknowledgments}

The authors acknowledge the financial support from the Boeing company

\section{References}

\footnotetext{
${ }^{1}$ M. Abramowitz and (Eds.) I.A. Stegun. 9.6 in Handbook of Mathematical Functions with Formulas, Graphs, and Mathematical Tables, 9th printing. New York: Dover, 1972.

${ }^{2}$ E.C. Aifantis. Update on a class of gradient theories. Mech. Mater., 35(3-6):259-280, 2003.

${ }^{3}$ Harm Askes and Elias C. Aifantis. Gradient elasticity in statics and dynamics: An overview of formulations, length scale identification procedures, finite element implementations and new results. International Journal of Solids and Structures, 48 (13):1962-1990, 2011.

${ }^{4}$ Z.P. Bazant and Ta-Peng Chang. Instability of nonlocal continuum and strain averaging. Journal of Engineering Mechanics, 110(10):1441-1450, 1984.

${ }^{5}$ Z.P. Bazant and M. Jirásek. Nonlocal integral formulations of plasticity and damage: survey of progress. J. Eng. Mech. ASCE, 128(11):11191149, 2002.

${ }^{6}$ R. N. Bracewell. In The Fourier Transform and Its Applications, 3rd ed. New York: McGraw-Hill, 1999.

${ }^{7}$ A.C. Eringen. On differential equations of nonlocal elasticity and solutions of screw dislocation and surface waves. Journal of Applied Physics, 54(9):4703-4710, 1983.

${ }^{8} \mathrm{~S}$. Ghosh, V. Sundararaghavan, and A.M. Waas. Construction of multi-dimensional isotropic kernels for nonlocal elasticity based on phonon dispersion data Manuscript under review, 2013.

${ }^{9}$ B. Osgood. The Fourier Transform and its Applications. Stanford University, 2009.

${ }^{10}$ R.C. Picu. On the functional form of non-local elasticity kernels. Journal of the Mechanics and Physics of Solids, 50(9): 1923-1939, 2002.

${ }^{11}$ Castrenze Polizzotto. Nonlocal elasticity and related variational principles. International Journal of Solids and Structures, 38(4243):7359 - 7380, 2001.

${ }^{12}$ P.K. Sahoo. Circularly symmetric separable functions are gaussian. Applied Mathematics Letters, 3(3):111 - 113 , 1990.

${ }^{13} \mathrm{~V}$. Sundararaghavan and Anthony Waas. Non-local continuum modeling of carbon nanotubes: Physical interpretation of non-local kernels using atomistic simulations. Journal of the Mechanics and Physics of Solids, 59(6):1191-1203, 2011.
} 